\title{
Quantitative Identification of Adulterated Sichuan Pepper Powder by Near-Infrared Spectroscopy Coupled with Chemometrics
}

\author{
Xi-Yu Wu, ${ }^{1,2}$ Shi-Ping Zhu, ${ }^{1}$ Hua Huang, ${ }^{1}$ and Dan $\mathrm{Xu}^{2}$ \\ ${ }^{1}$ College of Engineering and Technology, Southwest University, Chongqing 400716, China \\ ${ }^{2}$ College of Food Science, Southwest University, Chongqing 400716, China \\ Correspondence should be addressed to Shi-Ping Zhu; zspswu@126.com
}

Received 13 March 2017; Accepted 27 April 2017; Published 17 May 2017

Academic Editor: Giorgia Greco

Copyright (C) $2017 \mathrm{Xi}-Y u$ Wu et al. This is an open access article distributed under the Creative Commons Attribution License, which permits unrestricted use, distribution, and reproduction in any medium, provided the original work is properly cited.

\begin{abstract}
Sichuan pepper is a traditional and important flavoring of Chinese cuisine. It has attracted increasing interest in recent years owning to its unique taste and aroma. However, some cheap adulterants have been illegally found in Sichuan pepper powder in the market due to merchants trying to cut costs and gain an extra profit. In order to determine the compositions of Sichuan pepper powder quickly and effectively, a direct detection method using near-infrared (NIR) spectroscopy has been developed. 462 samples of adulterated Sichuan pepper powder mixed with different amounts of wheat bran, rice bran, corn flour, and rosin powder were studied. The NIR spectra data was studied using partial least squares (PLS) analysis. The method was found to be capable of predicting the compositions of adulterated Sichuan pepper powder. The determination coefficients of prediction set $\left(R_{\mathrm{p}}{ }^{2}\right)$ with the best pretreatments were 0.971 for Sichuan pepper powder, 0.948 for rice bran, 0.969 for wheat bran, 0.967 for corn flour, and 0.994 for rosin powder, respectively. The standard errors of prediction (SEP) were $2.81 \%, 2.38 \%, 3.19 \%, 2.46 \%$, and $1.10 \%$, respectively. The results showed that NIR spectroscopy with chemometrics is a rapid and nondestructive tool for the quantitative analysis of adulterated Sichuan pepper powder.
\end{abstract}

\section{Introduction}

Sichuan pepper, pericarp of Zanthoxylum bungeanum Maxim. and Zanthoxylum schinifolium Sieb. et Zucc. [1], is one of the most important spices in many Asian dishes. Particularly in China, it is known as "one of the eight famous condiments" and is widely used in Sichuanese cuisine [2]. Thanks to the increasing popularity of Chinese cuisine in many other countries of the world, Sichuan pepper is becoming a more and more widely used condiment. Sichuan pepper is also used as a traditional Chinese herbal medicine for the treatment of vomiting, toothache, stomachache, prurigo, and abdominal pain owing to roundworm [3-5]. Researchers have found that the main chemical components of Sichuan pepper are alkaloids, alkylamides, neolignans, volatile oil, and coumarin. These compounds possess several types of biological activities such as anti-inflammatory, analgesic, antibiotic, and anthelminthic activities [6-8].
For better seasoning and more ease of use, Sichuan pepper is usually ground into a powder before selling. However, because of the higher price of the Sichuan pepper powder, various cheaper adulterations have been added by sellers to gain illegal profits. Some of these adulterations have been found to be harmful to the human body and could result in potential health problems. Therefore, these behaviors could not only influence concerned consumers because of the potential health risks, but also affect normal market activities. Because of this background, effective and quick detection methods for adulterations are urgently needed. The commonly used adulterations have been found to be wheat bran, corn flour, rice bran, rosin powder, or traditional Chinese medicine residue [9]. The reported identification techniques of the potential adulterants are sensory recognition, microscopic recognition $[9,10]$, and iodide test for starchy material identification [10]. Sensory recognition is able to distinguish the adulterations according to their color, 
TABLE 1: 30 species of Sichuan pepper used in this study.

\begin{tabular}{lcc}
\hline Producing area & Number & Species \\
\hline Zigong & 1 & Zanthoxylum bungeanum Maxim. (red Sichuan pepper) \\
Maowen & 2 & Zanthoxylum bungeanum Maxim. (red Sichuan pepper) \\
Chengdu & 1 & Zanthoxylum bungeanum Maxim. (red Sichuan pepper) \\
Hanyuan & 2 & Zanthoxylum bungeanum Maxim. (red Sichuan pepper) \\
Hanyuan & 1 & Zanthoxylum schinifolium Sieb. et Zucc. (green Sichuan pepper) \\
Qingchuan & 1 & Zanthoxylum schinifolium Sieb. et Zucc. (green Sichuan pepper) \\
Jinyang & 1 & Zanthoxylum schinifolium Sieb. et Zucc. (green Sichuan pepper) \\
Chongqing & 4 & Zanthoxylum bungeanum Maxim. (red Sichuan pepper) \\
Chongqing & 3 & Zanthoxylum schinifolium Sieb. et Zucc. (green Sichuan pepper) \\
Jiangjin & 1 & Zanthoxylum schinifolium Sieb. et Zucc. (green Sichuan pepper) \\
Leling & 1 & Zanthoxylum bungeanum Maxim. (red Sichuan pepper) \\
Leling & 1 & Zanthoxylum schinifolium Sieb. et Zucc. (green Sichuan pepper) \\
Hancheng & 3 & Zanthoxylum bungeanum Maxim. (red Sichuan pepper) \\
Zhenfeng & 1 & Zanthoxylum bungeanum Maxim. (red Sichuan pepper) \\
Guanling & 2 & Zanthoxylum schinifolium Sieb. et Zucc. (green Sichuan pepper) \\
Kunming & 1 & Zanthoxylum bungeanum Maxim. (red Sichuan pepper) \\
Kunming & 2 & Zanthoxylum schinifolium Sieb. et Zucc. (green Sichuan pepper) \\
Zhaotong & 2 & Zanthoxylum schinifolium Sieb. et Zucc. (green Sichuan pepper) \\
\hline
\end{tabular}

smell, and taste, but this method depends on experience and is hard to quantify. Microscopic recognition requires sample pretreatment and has a complicated operation. The iodide test is only effective in identifying starchy adulterations. Therefore, none of the above methods can give a quick, accurate, and quantitative determination on the adulterants of Sichuan pepper powder to satisfy the practical need.

Near-infrared (NIR) spectroscopy has been developed in recent years as an attractive technique for quantification of various substances in food samples because it is fast, nondestructive, and inexpensive and only needs a small amount of sample with minimum pretreatment [11, 12]. The wavelength range of NIR is between $800 \mathrm{~nm}$ and $2500 \mathrm{~nm}$ $\left(12500 \sim 4000 \mathrm{~cm}^{-1}\right)$. The absorptions appearing in the range of 800 to $1800 \mathrm{~nm}\left(12500 \sim 5500 \mathrm{~cm}^{-1}\right)$ are molecular absorptions of overtone, while those in the range of 1800 to $2500 \mathrm{~nm}$ $\left(5500 \sim 4000 \mathrm{~cm}^{-1}\right)$ are combination bands of fundamental vibrational transitions mainly associated with $\mathrm{C}-\mathrm{H}, \mathrm{O}-\mathrm{H}$, and $\mathrm{N}-\mathrm{H}$ functional groups of the testing samples. NIR bands are the result of complex anharmonicity vibrational motion of these chemical bonds [13, 14]. Combined with the chemometric methods, NIR spectroscopy is becoming a powerful tool for quantitative and qualitative determination of food components [15-17]. Therefore, these tools can be easily applied to evaluate food quality and determine authenticity. Currently, NIR spectroscopy has been used to identify adulterations in puerarin powder, chocolate, and edible oils to name a few [18-20]. However, no studies have been found so far concerning the identification and authentication of Sichuan pepper by NIR spectroscopy, particularly when Sichuan pepper powder is employed. Therefore, this study combined spectroscopy with partial least squares (PLS) to identify four adulterations, that is, wheat bran, rice bran, corn flour, and rosin powder, in Sichuan pepper powder. The aim was also to establish an authentic quantitative discrimination model to provide technical support for market management of Sichuan pepper powder.

\section{Materials and Methods}

2.1. Samples. Thirty samples of Sichuan pepper with obvious differences in origin, character, and quality were collected from different regions in China (Table 1). All Sichuan pepper samples were milled into powder through a high-speed universal grinder (Pulverisette 14, Fritsch Co., Idar-Oberstein, Germany, $0.5 \mathrm{~mm}$ standard sieve). Four adulterations (wheat bran, rice bran, corn, and rosin) were separately milled and sieved. Next, the adulterations were accurately weighed by an electronic analytical balance (FA2004A, Shanghai Jingtian Electronic Instrument Co., Ltd., Shanghai, China, precision $0.0001 \mathrm{~g}$ ) and were added to red Sichuan pepper powder and green Sichuan pepper powder, respectively, with different proportions (1 54 wt./wt.\% and 1 wt./wt.\% intervals). After mixing, 432 samples were obtained. All pure and adulterated Sichuan pepper powder samples (462 in total) were packed and numbered under the same conditions. Samples were stored at $25^{\circ} \mathrm{C}$ before testing. NIR measurements of all samples were performed in 12 hours.

2.2. NIR Spectroscopy Measurement. The spectra were collected at $25^{\circ} \mathrm{C}$ using a MPA near-infrared spectrometer (Bruker Optik GmbH, Ettlingen, Germany) equipped with a $\mathrm{PbS}$ detector, an integrating sphere, and a quartz cup. $10 \mathrm{~g}$ samples were filled into the quartz cup and scanned directly. Diffuse reflection light detection was used and all the 
spectra were recorded at the resolution of $8 \mathrm{~cm}^{-1}$, performing 32 scans for each spectrum from 3800 to $12500 \mathrm{~cm}^{-1}$. The spectrum of each sample was taken in triplicate and the mean spectrum of each sample was calculated for further statistical analysis.

Spectral data acquisition was achieved with software OPUS7.0 (Bruker Optik GmbH, Ettlingen, Germany), installed under a Windows 7 operating system.

2.3. Chemometric and Statistical Analysis. To reduce the dimension of the data matrix and to compress the information into a few interpretable variables, principal component analysis (PCA) was adopted as an exploratory method in this experiment. The variables are called principal components (PCs), which are linear combinations of the original variables [21]. The first three PCs (PC1, PC2, and PC3) give useful information on the score plot such that similar samples are clustered close to each other [11].

By analyzing the relationship between the NIR wavelength information and adulterations ratios of Sichuan pepper powder, calibration models were performed by partial least squares (PLS) regression. To eliminate the baseline drift and random noise of spectral signal, three mathematical pretreatments (no mathematical processing, first derivative, and second derivative) were tested. To eliminate the impact of particle size distribution of samples caused by uneven scattering, four scattering corrections (no scattering processing, multiplicative scatter corrections (MSC), standard normal variate (SNV), and maximum/minimum normalized) were tested. The best model was selected for the proportions of Sichuan pepper powder, wheat bran, rice bran, corn flour, and rosin powder on the basis of the highest correlation coefficients and the lowest standard errors of calibration and prediction, respectively [22].

In order to select the optimal number of factors and to avoid overfitting, cross-validation was employed [16, 17]. In this method, one sample of the calibration set was used to check the results of the calibration model, which was constructed by the remaining samples. The model was repeated until every sample had finished being validated. Calibration errors were combined in a standard error of cross-validation (SEC) of the calibration set [23]. The determination coefficient of the calibration set $\left(R_{\mathrm{c}}{ }^{2}\right)$ was considered as the best single estimate for the prediction capability of the equation $[16,17]$. Once the model was obtained, external validation was performed without any samples in the calibration set. Similar to the above-mentioned method, the standard error of prediction (SEP) and the determination coefficient of external validation set $\left(R_{\mathrm{p}}{ }^{2}\right)$ reflected the prediction ability of the model [24]. Furthermore, RPD was the ratio of standard deviation of reference data in prediction set to SEP. A RPD value above 3.0 was regarded as satisfactory for screening and value above 5.0 was suitable for quality control analysis $[25,26]$.

In data processing, PCA and PLS were implemented in Matlab R2015a (MathWorks, Inc., Natick, Massachusetts, USA) under Windows 10.

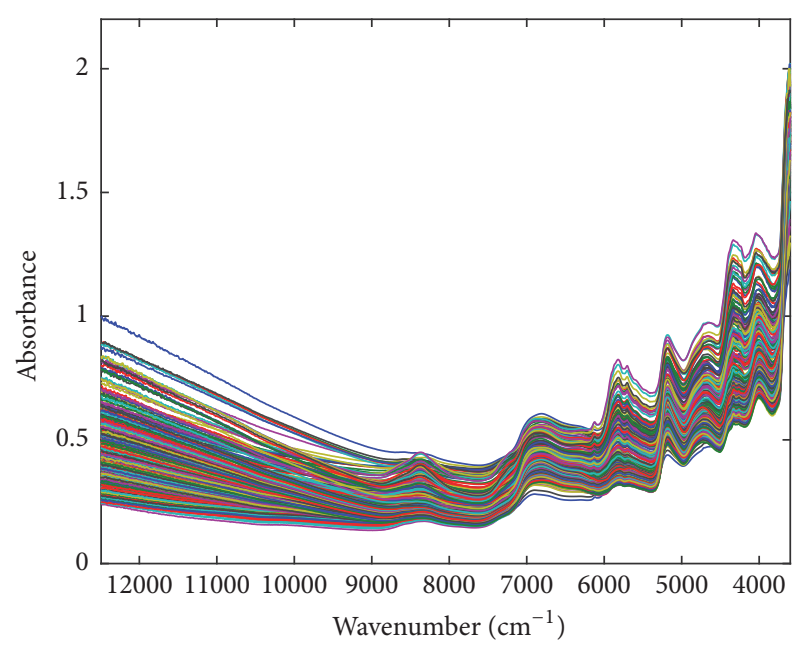

FIGURE 1: NIR spectra of 462 samples.

2.4. Calibration Set and Prediction Set. In this experiment, the data set was composed of 462 samples, which were divided into two parts: calibration set (347 samples) and prediction set (115 samples). The calibration set was used to build the models, while the prediction set was used for evaluating the actual predictive ability of the models [11]. To avoid bias, all the samples were arranged from high to low according to the Sichuan pepper powder content. And the calibration set and prediction set were partitioned as follows: for every four samples, three spectra were selected as the calibration set in sequence, while the remaining one was used as the prediction set.

\section{Results and Discussion}

3.1. NIR Spectra. Figure 1 shows the NIR spectra of 462 samples in this study. The peak shapes and positions of all the samples' NIR spectra are relatively similar in appearance. It is difficult to identify the adulteration samples directly through the spectra. Therefore, the chemometric methods should be adopted in order to establish the detection models to identify the adulterated Sichuan pepper powder.

The average spectra of wheat bran, rice bran, corn flour, rosin powder, and Sichuan pepper powder are presented in Figure 2. There are significant differences between Sichuan pepper powder and the four adulterations in the spectra, attributed to the tremendous differences of their constituents. These spectral characteristics, therefore, provide a wealth of information to the quantitative analysis of adulterated Sichuan pepper powder.

3.2. Principal Component Analysis. Principal component analysis (PCA) was performed on all the spectra data in order to reveal the main features and clusters of samples [27]. The score distribution of PC1, PC2, and PC3 for adulterated Sichuan pepper powder in Figure 3 shows that there is some 


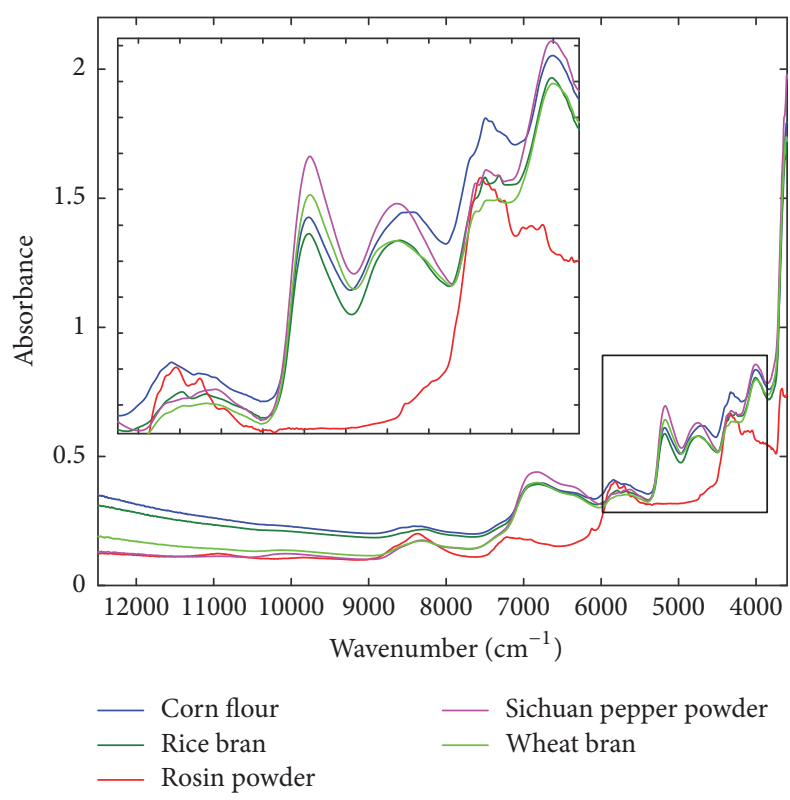

FIGURE 2: NIR spectra of Sichuan pepper powder, wheat bran, rice bran, corn flour, and rosin powder.

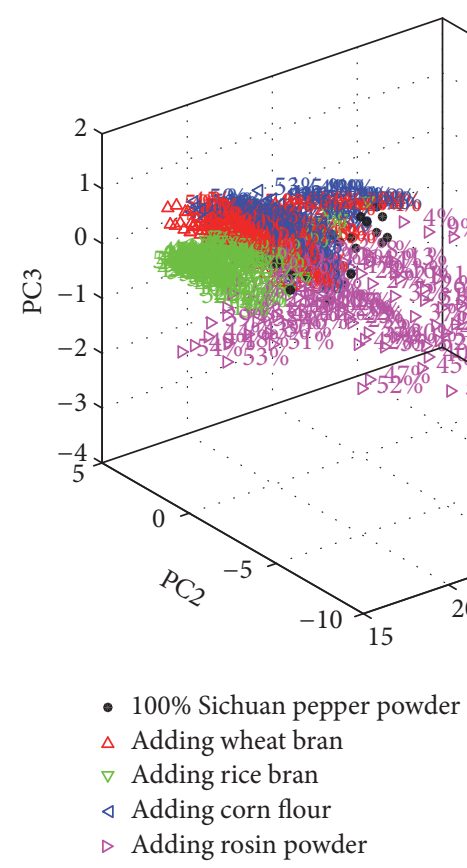

Figure 3: PCA on the calibration and prediction sets of adulterated Sichuan pepper powder samples.

information, characteristic groupings, related to the varieties and content of samples [28]. With the increasing content of Sichuan pepper powder, the points corresponding to the adulterations of wheat bran, rice bran, corn flour, and rosin powder, respectively, get closer to the points of pure Sichuan pepper powder. The distribution areas of different adulterated samples are more apparent and the points with similar adulterated proportions of the same samples are closer relatively. Therefore, according to the spectral features of adulterated Sichuan pepper powder, a positive recognition of them can be done because of their differences in compositional traits.

3.3. NIR Determination of Adulterated Sichuan Pepper Powder. The calibration process was performed with the spectra of calibration sets (347 samples) and their corresponding proportions of adulterated components. To study the effects of different spectral preprocessing on the calibration results, PLS was adopted to establish the calibration model. Firstly, in the full spectrum range $\left(3800 \sim 12500 \mathrm{~cm}^{-1}\right)$, calibration models were established with several mathematical pretreatments and scattering corrections. Then, to evaluate the predictive ability of the calibration models, the prediction set (115 samples) was used. The calculated value of the statistical parameters of calibration and prediction set for each component of adulterated Sichuan pepper powder is shown in Table 2.

Revealed by Table 2, mathematical pretreatment to the calibration result was important and the differences were relatively distinct to forecast result when using different pretreatments. Based on the analysis of relative error of RPD, "MSC" was chosen as the optimal spectral pretreatment method for recognition of Sichuan pepper powder and rice bran. "First derivative $+\mathrm{SNV}$ " was chosen for wheat bran and corn flour, while "SNV" was chosen for rosin powder. With the best pretreatment above, the best determination coefficient of calibration set $\left(R_{\mathrm{c}}{ }^{2}\right)$ was $0.984,0.974,0.944$, 0.982 , and 0.996 for Sichuan pepper powder, wheat bran, rice bran, corn flour, and rosin powder, respectively, while the determination coefficient of prediction set $\left(R_{\mathrm{p}}{ }^{2}\right)$ was 0.971 , $0.969,0.948,0.967$, and 0.994 for the samples, respectively. Figure 4 shows the correlation of the values with respect to those predicted by NIR spectrometry for the content of adulterated Sichuan pepper powder. As calculated from Figure 4, there is a good linear correlation and the minimum relative error (RPD) of validation is 4.38. Five models have high prediction accuracy; therefore, within the scope of the full spectra of NIR spectroscopy, the calibration model is ideal.

\section{Conclusions}

The results showed that the NIR technique combined with PLS regression analysis can be used to quantitatively identify adulterations including wheat bran, rice bran, corn flour, and rosin powder in Sichuan pepper powder nondestructively. The contents of wheat bran, rice bran, corn flour, and rosin powder in adulterated Sichuan pepper powder could be discriminated directly based on the spectra data and require no pretreatment of samples. Therefore, as a simple, quick, reliable, and nondestructive technique, NIR may be suitable for the quality detection of packaged seasoning at a low cost, particularly when robust NIR equipment is developed. 


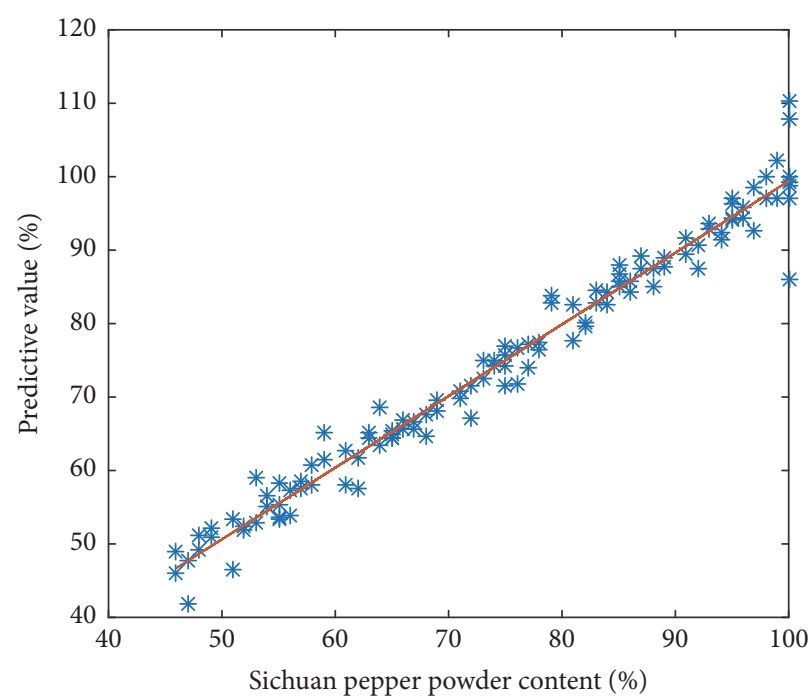

(a) Forecast figure of Sichuan pepper powder content of prediction set

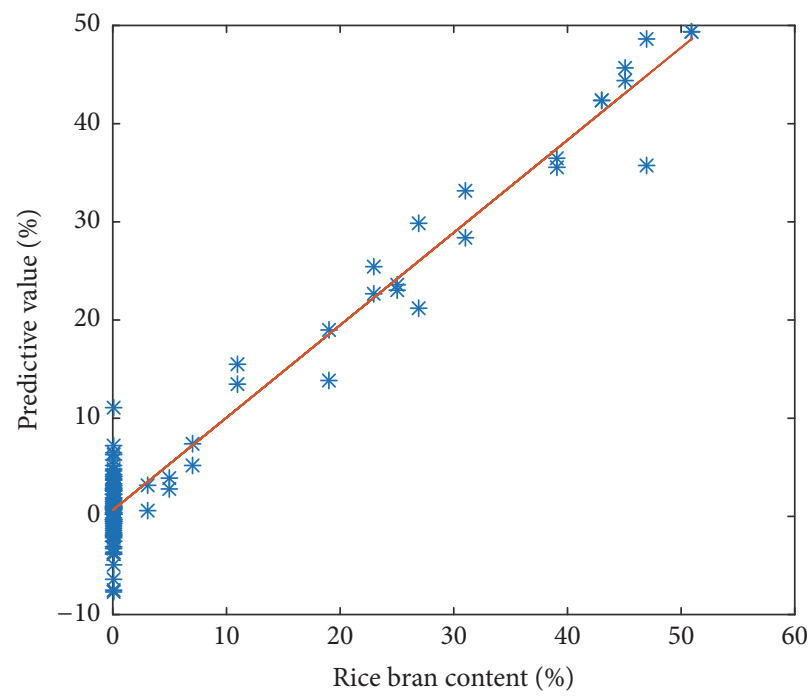

(c) Forecast figure of rice bran content of prediction set

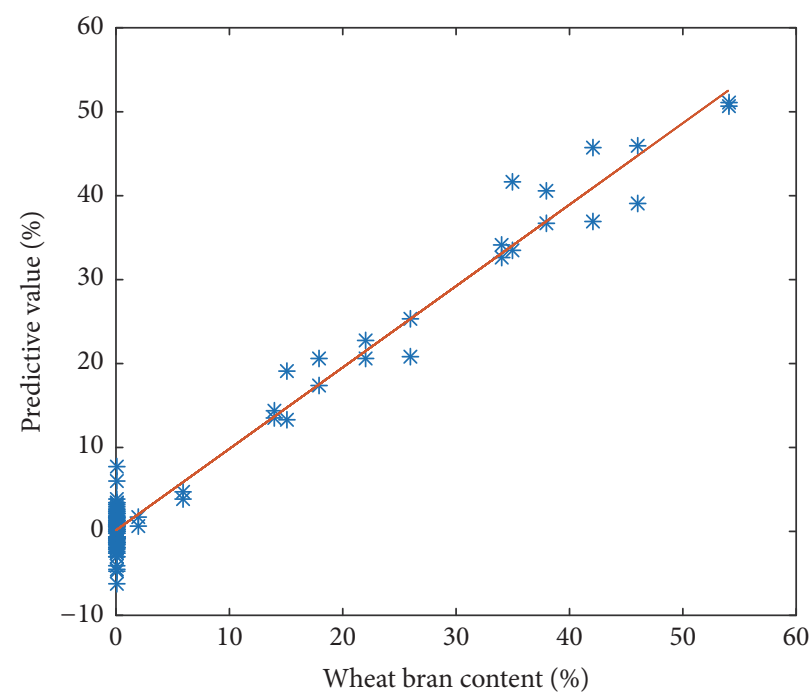

(b) Forecast figure of wheat bran content of prediction set

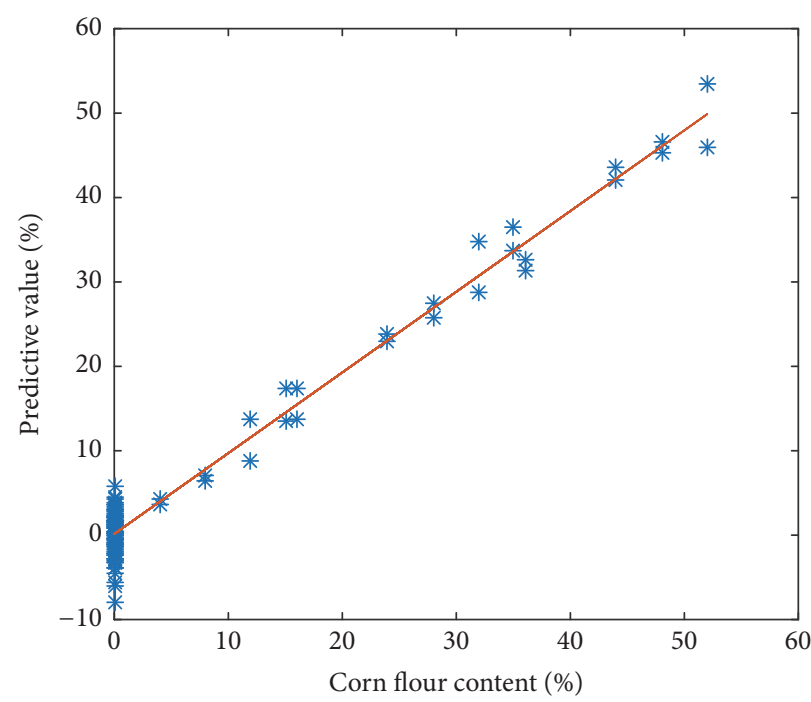

(d) Forecast figure of corn flour content of prediction set

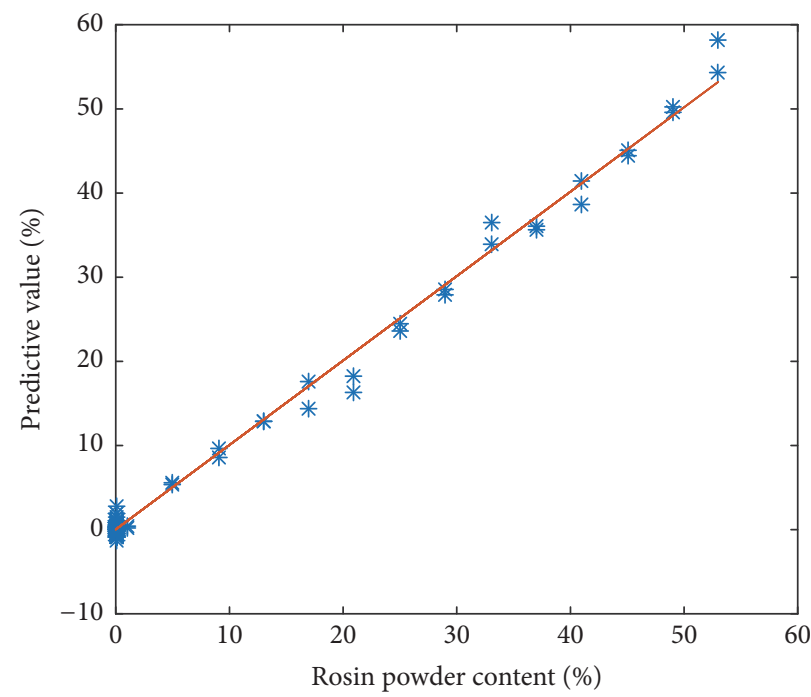

(e) Forecast figure of rosin powder content of prediction set

FigURE 4: Prediction results for components of adulterated Sichuan pepper powder in prediction set using NIR spectroscopy. 
TABLE 2: Results of PLS calibration models with different mathematical pretreatments on spectra.

\begin{tabular}{|c|c|c|c|c|c|c|c|}
\hline \multirow[t]{2}{*}{ Components } & \multirow[t]{2}{*}{ Mathematical pretreatment } & \multirow[t]{2}{*}{$\begin{array}{l}\text { Number of } \\
\text { principal } \\
\text { components }\end{array}$} & \multicolumn{5}{|c|}{$\begin{array}{l}\text { rediction } \mathrm{s} \\
(n=115)\end{array}$} \\
\hline & & & $R_{\mathrm{c}}^{2}$ & SEC/\% & $R_{\mathrm{p}}^{2}$ & SEP/\% & $\mathrm{RPD}$ \\
\hline \multirow{6}{*}{ Sichuan pepper powder } & No processing & 10 & 0.961 & 3.29 & 0.929 & 4.38 & 3.75 \\
\hline & SNV & 10 & 0.984 & 2.10 & 0.970 & 2.87 & 5.73 \\
\hline & $M S C$ & 10 & 0.984 & 2.12 & 0.971 & 2.81 & 5.84 \\
\hline & Maximum/minimum normalized & 10 & 0.974 & 2.67 & 0.962 & 3.21 & 5.12 \\
\hline & First derivative $+\mathrm{SNV}$ & 9 & 0.969 & 2.90 & 0.967 & 2.99 & 5.51 \\
\hline & Second derivative + MSC & 9 & 0.969 & 2.92 & 0.936 & 4.15 & 3.98 \\
\hline \multirow{6}{*}{ Wheat bran } & No processing & 10 & 0.965 & 2.64 & 0.846 & 5.29 & 2.55 \\
\hline & SNV & 10 & 0.977 & 2.11 & 0.912 & 3.99 & 3.38 \\
\hline & MSC & 9 & 0.977 & 2.13 & 0.909 & 4.24 & 3.18 \\
\hline & Maximum/minimum normalized & 10 & 0.973 & 2.32 & 0.899 & 4.27 & 3.16 \\
\hline & First derivative $+S N V$ & 10 & 0.974 & 2.27 & 0.969 & 2.38 & 5.66 \\
\hline & Second derivative + MSC & 10 & 0.959 & 2.84 & 0.900 & 4.26 & 3.16 \\
\hline \multirow{6}{*}{ Rice bran } & No processing & 10 & 0.897 & 4.44 & 0.860 & 5.21 & 2.68 \\
\hline & SNV & 10 & 0.945 & 3.25 & 0.937 & 3.49 & 4.00 \\
\hline & MSC & 10 & 0.944 & 3.29 & 0.948 & 3.19 & 4.38 \\
\hline & Maximum/minimum normalized & 10 & 0.928 & 3.71 & 0.940 & 3.41 & 4.09 \\
\hline & First derivative $+\mathrm{SNV}$ & 10 & 0.928 & 3.71 & 0.940 & 3.41 & 4.09 \\
\hline & Second derivative + MSC & 9 & 0.897 & 4.44 & 0.797 & 6.27 & 2.23 \\
\hline \multirow{6}{*}{ Corn flour } & No processing & 10 & 0.979 & 2.05 & 0.916 & 3.89 & 3.46 \\
\hline & SNV & 10 & 0.986 & 1.66 & 0.944 & 3.18 & 4.23 \\
\hline & MSC & 9 & 0.987 & 1.59 & 0.946 & 3.13 & 4.29 \\
\hline & Maximum/minimum normalized & 9 & 0.984 & 1.75 & 0.930 & 3.54 & 3.80 \\
\hline & First derivative $+S N V$ & 10 & 0.982 & 1.89 & 0.967 & 2.46 & 5.47 \\
\hline & Second derivative + MSC & 10 & 0.969 & 2.46 & 0.922 & 3.76 & 3.58 \\
\hline \multirow{6}{*}{ Rosin powder } & No processing & 10 & 0.984 & 1.75 & 0.989 & 1.45 & 9.69 \\
\hline & $S N V$ & 10 & 0.996 & 0.83 & 0.994 & 1.10 & 12.8 \\
\hline & MSC & 10 & 0.996 & 0.87 & 0.995 & 1.03 & 13.7 \\
\hline & Maximum/minimum normalized & 9 & 0.991 & 1.28 & 0.989 & 1.50 & 9.38 \\
\hline & First derivative $+\mathrm{SNV}$ & 7 & 0.993 & 1.13 & 0.992 & 1.24 & 11.4 \\
\hline & Second derivative + MSC & 6 & 0.993 & 1.20 & 0.989 & 1.49 & 9.41 \\
\hline
\end{tabular}

\section{Disclosure}

This article does not contain any studies with human participants or animals performed by any of the authors.

\section{Conflicts of Interest}

The authors have no conflicts of interest regarding the publication of this paper.

\section{Acknowledgments}

This study was financially supported by the National Natural Science Foundation of China (Grants nos. 30671198 and 31071319) and the Fundamental Research Funds for the Central Universities (Grant no. XDJK2015C137).

\section{References}

[1] K. Mizutani, Y. Fukunaga, O. Tanaka et al., "Amides from huajiao, pericarps of Zanthoxylum bungeanum MAXIM," Chemical and Pharmaceutical Bulletin, vol. 36, no. 7, pp. 2362-2365, 1988.

[2] X. Yang, "Aroma constituents and alkylamides of red and green huajiao (Zanthoxylum bungeanum and Zanthoxylum schinifolium)," Journal of Agricultural and Food Chemistry, vol. 56, no. 5, pp. 1689-1696, 2008.

[3] The Pharmacopoeia Committee of the Ministry of Health of the People's Republic of China, The People's Republic of China Pharmacopoeia, People's Medical Publishing House, Chemical Industry Press, Beijing, China, 1990.

[4] C. J. Huang, "Rutaceae, Dicotyledoneae, Angiospermae," in Flora Reipublicae Sinicae, vol. 43, pp. 13-53, Science Press, Beijing, China, 1997. 
[5] Q. Xiong, D. Shi, and M. Mizuno, "Flavonol glucosides in pericarps of Zanthoxylum bungeanum," Phytochemistry, vol. 39, no. 3, pp. 723-725, 1995.

[6] J. S. Negi, V. K. Bisht, A. K. Bhandari, P. Singh, and R. C. Sundriyal, "Chemical constituents and biological activities of the genus Zanthoxylum: a review," Afr. J. Pure Appl. Chem, vol. 5, pp. 412-416, 2011.

[7] Y. Gong, Y. Huang, L. Zhou et al., "Chemical composition and antifungal activity of the fruit oil of Zanthoxylum bungeanum maxim. (Rutaceae) from China," Journal of Essential Oil Research, vol. 21, no. 2, pp. 174-178, 2009.

[8] W. Tang, Q. Xie, J. Guan, S. Jin, and Y. Zhao, "Phytochemical profiles and biological activity evaluation of Zanthoxylum bungeanum Maxim seed against asthma in murine models," Journal of Ethnopharmacology, vol. 152, no. 3, pp. 444-450, 2014.

[9] J. Xu and Y. J. Li, "Inspection method of 125-share adulterated condiment powder and the result analysis," China Brewing, vol. 5, pp. 31-33, 2004.

[10] Y. J. Li, "Types and proportions of adulterations in six kinds of condiments," China Condiment, vol. 8, pp. 81-83, 2008.

[11] E. Teye, X.-Y. Huang, W. Lei, and H. Dai, "Feasibility study on the use of Fourier transform near-infrared spectroscopy together with chemometrics to discriminate and quantify adulteration in cocoa beans," Food Research International, vol. 55, pp. 288-293, 2014.

[12] A. Subramanian, V. B. Alvarez, W. J. Harper, and L. E. Rodriguez-Saona, "Monitoring amino acids, organic acids, and ripening changes in Cheddar cheese using Fourier-transform infrared spectroscopy," International Dairy Journal, vol. 21, no. 6, pp. 434-440, 2011.

[13] J. S. Shenk and M. O. Westerhaus, "The application of near infrared reflectance spectroscopy (NIRS) to forage analysis," in Forage Quality, Evaluation, and Utilization, C. Fahey, Ed., ACSESS Publications, pp. 406-449, American Society of Agronomy, Crop Science Society of America, Soil Science Society of America, Wis, USA, 1994.

[14] L. E. Rodriguez-Saona and M. E. Allendorf, "Use of FTIR for rapid authentication and detection of adulteration of food," Annual Review of Food Science and Technology, vol. 2, pp. 467483, 2011.

[15] M. Bevilacqua, R. Bucci, S. Materazzi, and F. Marini, "Application of near infrared (NIR) spectroscopy coupled to chemometrics for dried egg-pasta characterization and egg content quantification," Food Chemistry, vol. 140, no. 4, pp. 726-734, 2013.

[16] M. I. González Martín, G. Wells Moncada, S. Fischer, and O. Escuredo, "Chemical characteristics and mineral composition of quinoa by near-infrared spectroscopy," Journal of the Science of Food and Agriculture, vol. 94, no. 5, pp. 876-881, 2014.

[17] M. I. González-Martín, G. W. Moncada, C. González-Pérez et al., "Chilean flour and wheat grain: tracing their origin using near infrared spectroscopy and chemometrics," Food Chemistry, vol. 145, pp. 802-806, 2014.

[18] J. Chen, J. Liu, Y. Q. Ma, S. P. Zhu, and G. H. Zhao, "Rapid detection of Kudzu starch adulteration by near-infrared diffuse reflectance spectroscopy," Food Science, vol. 35, no. 8, pp. 133136, 2014.

[19] T. Ma, H. Kobori, N. Katayama, and S. Tsuchikawa, "Nondestructive inspection of insects in chocolate using near infrared multispectral imaging," Journal of Near Infrared Spectroscopy, vol. 24, no. 4, pp. 391-397, 2016.
[20] N. Sinelli, L. Cerretani, V. D. Egidio, A. Bendini, and E. Casiraghi, "Application of near (NIR) infrared and mid (MIR) infrared spectroscopy as a rapid tool to classify extra virgin olive oil on the basis of fruity attribute intensity," Food Research International, vol. 43, no. 1, pp. 369-375, 2010.

[21] A. S. Luna, A. P. Da Silva, J. S. A. Pinho, J. Ferré, and R. Boqué, "Rapid characterization of transgenic and nontransgenic soybean oils by chemometric methods using NIR spectroscopy," Spectrochimica Acta - Part A: Molecular and Biomolecular Spectroscopy, vol. 100, pp. 115-119, 2013.

[22] I. González-Martín, C. González-Pérez, J. Hernández-Méndez, N. Alvarez-García, and J.-L. H. Andaluz, "On-line nondestructive determination of proteins and infiltrated fat in Iberian pork loin by near infrared spectrometry with a remote reflectance fibre optic probe," Analytica Chimica Acta, vol. 453, no. 2, pp. 281-288, 2002.

[23] P. C. Williams, A. M. C. Davies, and P. Williams, "Near infrared spectroscopy: the future waves," in Proceedings of the 7th International Conference on Near Infrared Spectroscopy, p. 742, NIR Publications, Montreal, Canada, 1996.

[24] I. Sone, R. L. Olsen, R. Dahl, and K. Heia, "Visible/near-infrared spectroscopy detects autolytic changes during storage of atlantic salmon (Salmo salar L.)," Journal of Food Science, vol. 76, no. 3, pp. S203-S209, 2011.

[25] O. Poungchompu, S. Saranwong, and S. Kawano, "Performance of a portable 263 NIR instrument "NIR-Gun" for determining raw milk compositions using transmittance," in Proceedings of the Second Asian NIR Spectroscopy, vol. 17, pp. 34-35, 2011.

[26] R. Du, K. Lai, Z. Xiao, Y. Shen, X. Wang, and Y. Huang, "Evaluation of the quality of deep frying oils with fourier transform near-infrared and mid-infrared spectroscop," Journal of Food Science, vol. 77, no. 2, pp. C261-C266, 2012.

[27] O. Galtier, N. Dupuy, Y. Le Dréau et al., "Geographic origins and compositions of virgin olive oils determinated by chemometric analysis of NIR spectra," Analytica Chimica Acta, vol. 595, no. 1-2, pp. 136-144, 2007.

[28] L. W. Mamani-Linares, C. Gallo, and D. Alomar, "Identification of cattle, llama and horse meat by near infrared reflectance or transflectance spectroscopy," Meat Science, vol. 90, no. 2, pp. 378-385, 2012. 

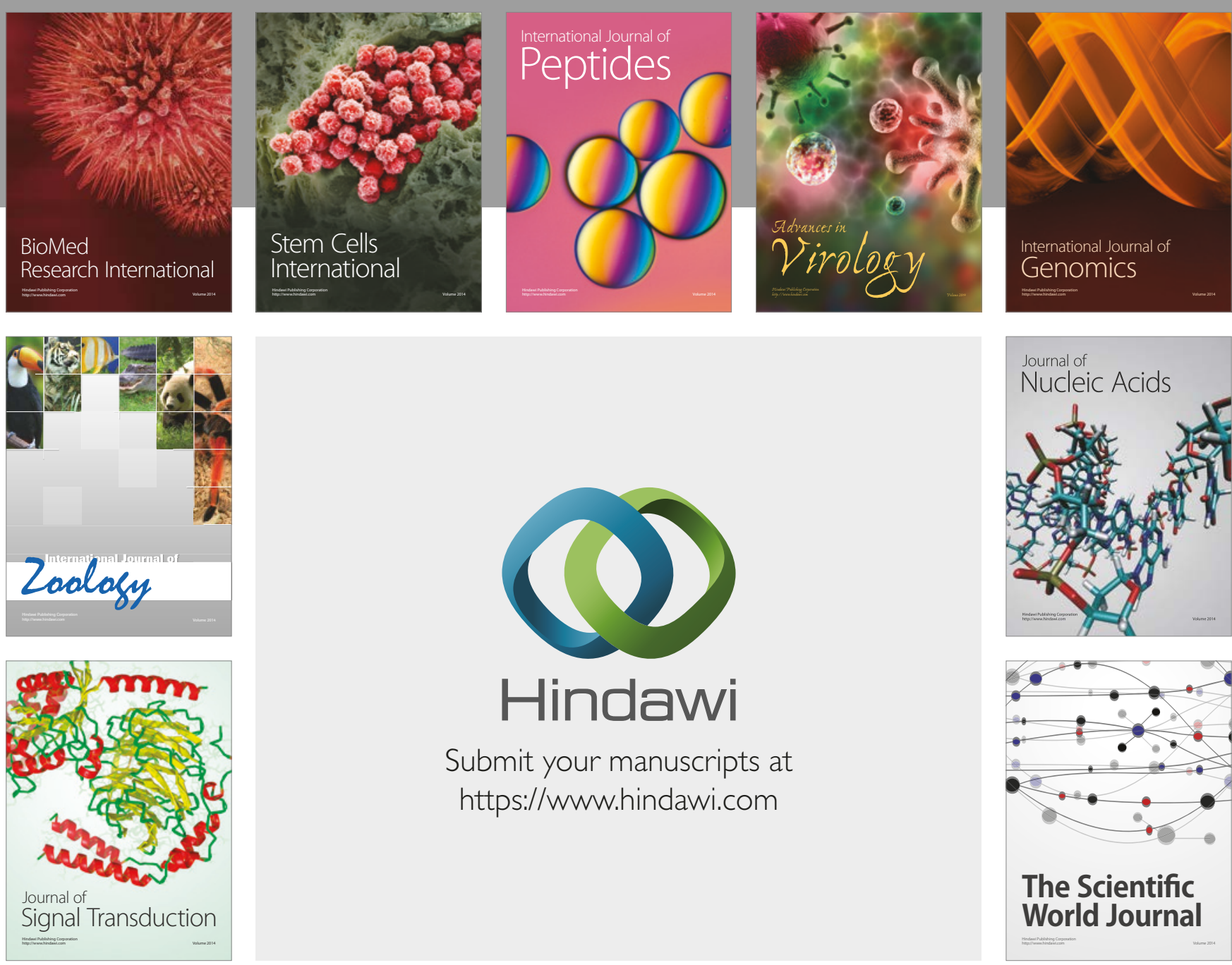

Submit your manuscripts at

https://www.hindawi.com
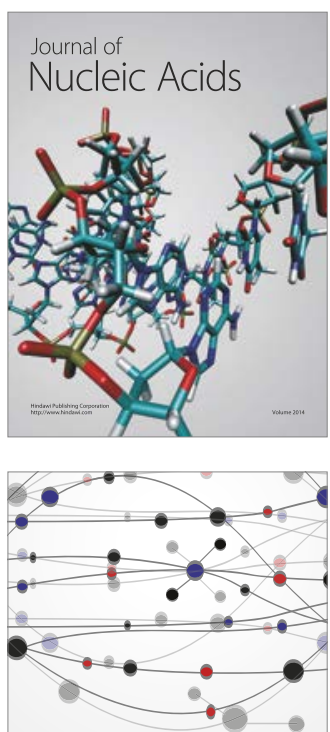

The Scientific World Journal

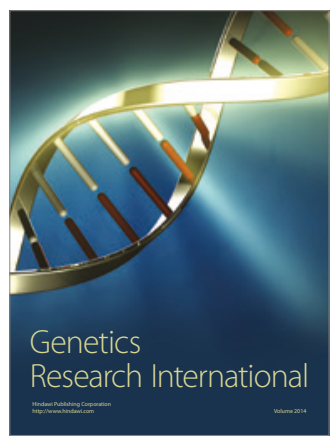

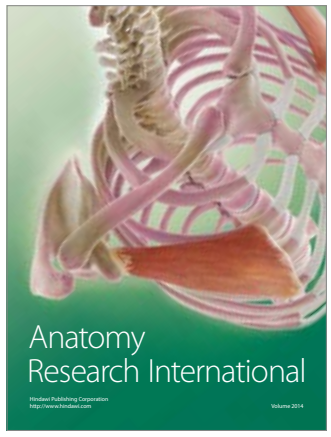

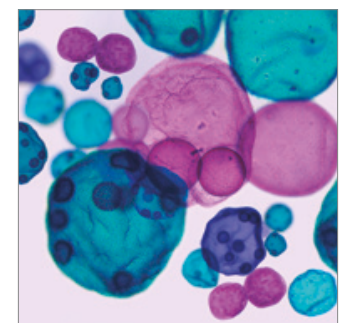

International Journal of Microbiology
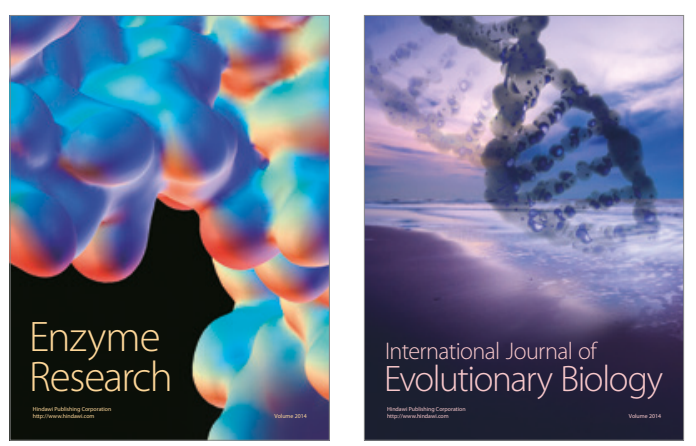
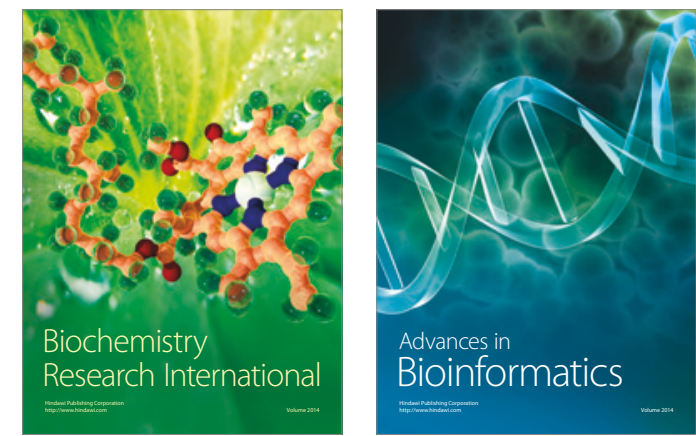

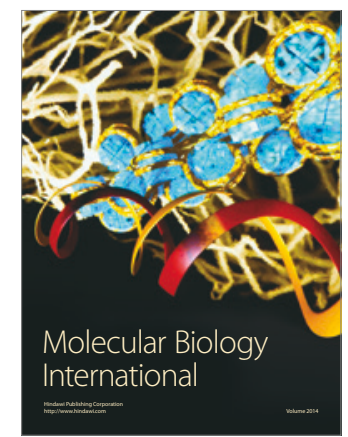

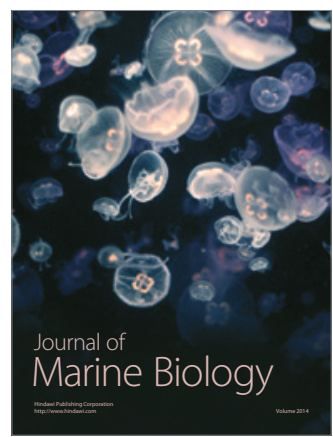

